\title{
Article
}

\section{Should comprehensive diagnosis include idiographic understanding?}

Thornton, Tim

Available at http://clok.uclan.ac.uk/3227/

Thornton, Tim ORCID: 0000-0002-0137-1554 (2008) Should comprehensive diagnosis include idiographic understanding? Medicine, health care, and philosophy, 11 (3). pp. 293-302. ISSN 1386-7423

It is advisable to refer to the publisher's version if you intend to cite from the work.

For more information about UCLan's research in this area go to

http://www.uclan.ac.uk/researchgroups/ and search for < name of research Group>.

For information about Research generally at UCLan please go to

http://www.uclan.ac.uk/research/

All outputs in CLoK are protected by Intellectual Property Rights law, including

Copyright law. Copyright, IPR and Moral Rights for the works on this site are retained by the individual authors and/or other copyright owners. Terms and conditions for use of this material are defined in the policies page.

\section{CLoK}

Central Lancashire online Knowledge www.clok.uclan.ac.uk

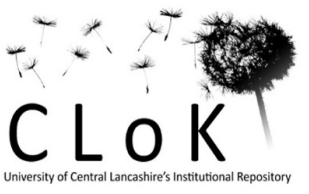


Full Title: Should comprehensive diagnosis include idiographic understanding?

Short title: Idiographic understanding

Authors (i.e. name as it should appear on the paper):

Tim Thornton

Position (i.e. job description as it should appear on the paper):

Tim Thornton

Professor of Philosophy and Mental Health

Address for correspondence:

Institute for Philosophy, Diversity and Mental Health

University of Central Lancashire

Preston

PR1 2HE

Other Contact Details:

phone 01772895412

fax 01772892964

email:

TThornton1@uclan.ac.uk

Key-words (not appearing in title):

Philosophy, psychiatry, comprehensive diagnosis, Windelband, nomothetic.

Word count (excluding title page but including references):

$\sim 6,600$

Character count (abstract):

Software used: Microsoft Word

Filename(s): 


\title{
Should comprehensive diagnosis include idiographic understanding?
}

\begin{abstract}
The World Psychiatric Association has emphasised the importance of idiographic understanding as a distinct component of comprehensive assessment but in introductions to the idea it is often assimilated to the notion of narrative judgement. This paper aims to distinguish between supposed idiographic and narrative judgement. Taking the former to mean a kind of individualised judgement, I argue that it has no place in psychiatry in part because it threatens psychiatric validity. Narrative judgement, by contrast, is a genuinely distinct complement to criteriological diagnosis but it is, nevertheless, a special kind of general judgement and thus can possess validity.
\end{abstract}

To do this I first examine the origin of the distinction between idiographic and nomothetic in Windelband's 1894 rectorial address. I argue that none of three ways of understanding that distinction is tenable. Windelband's description of historical methods, as a practical example, does not articulate a genuine form of understanding. A metaphysical distinction between particulars and general kinds is guilty of subscribing to the Myth of the Given. A distinction based on an abstraction of essentially combined aspects of empirical judgement cannot underpin a distinct empirical method. Furthermore, idiographic elements understood as individualised judgements threaten the validity of psychiatric diagnosis.

In the final part I briefly describe some aspects of the logic of narrative judgements and argue that in the call for comprehensive diagnosis, narrative rather than idiographic elements have an important role. Importantly, however, whilst directed towards individual subjects, narratives are framed in intrinsically general concepts and thus can aspire to validity.

Declaration of interests: none.

\section{Introduction}

There has been a recent growth in emphasis on the importance of idiographic understanding in psychiatric diagnosis and, more broadly, formulation. The World Psychiatric Association advocates the development of a 'comprehensive' model of diagnosis or assessment as part of its ongoing Institutional Program for Psychiatry for the Person. A WPA workgroup charged with formulating 'International Guidelines for Diagnostic Assessment' (IGDA) has published a guideline called 'Idiographic (Personalised) Diagnostic Formulation' which recommends an idiographic component alongside criteriological diagnosis. But these calls for an idiographic component risk blurring two motivations for, and two conceptions of, the kind of element that should be added to conventional criteriological diagnosis. In this paper I outline what these two conceptions are. Whilst both are directed at understanding individuals, one calls for a kind of individualised judgement, a notion I will attempt to explain. But it turns out to be a myth. The other, whilst aimed at understanding individuals, and genuinely distinct from criteriological psychiatric diagnosis, is not individualised but is general in a way that is distinct from criteriological diagnosis.

In fact, in recent pronouncements by supporters of the WPA, both these senses are run together. My aim in this paper is thus not to criticise the WPA's Program for Psychiatry for the Person and its call for judgement to recognise individual subjects or for psychiatric diagnosis, narrowly construed, to be augmented by a broader notion of psychiatric formulation. Nor is it to criticise their implicit suggestion that idiographic judgement should be construed as narrative judgement. Rather, my aim is to make this last implicit suggestion explicit. Idiographic judgement should be replaced by narrative judgement and the aim of capturing subjective experience should be construed as aided by narrative judgement which is, in an important sense, normative, rather than by the mythical notion of individualised idiographic judgement. This will help articulate what more is involved in formulation over diagnosis: not individualised judgement but narrative judgement. But narrative judgement, 
unlike idiographic judgement, turns out to be general.

To argue against the notion of individualised idiographic judgement will require a lengthy diversion via Windelband's rectorial address. This might seem unnecessary and excessive. After all, in psychology, a distinction between idiographic and nomothetic has been apparently unproblematically deployed since the time of the personality psychologist G.W. Allport [although see Hurlburt and Knapp 2006]. It refers to a distinction between qualitative research based on individual case studies and quantitative cohort-based research. But in the context of the WPA's Institutional Program for Psychiatry for the Person, the important question is precisely how is the individual to be represented in psychiatric diagnosis? What new element, if any, should be added to criteriological judgements? How, conceptually, might a qualitative approach differ from a quantitative approach? It is important to get the theoretical basis for a proposed change in emphasis in psychiatric diagnosis (or, rather, formulation) right and for this reason I will examine Windelband's explicit introduction carefully.

\section{Background}

The World Psychiatric Association's proposed Idiographic (Personalised) Diagnostic Formulation closely connects the idea of a comprehensive model of diagnosis with an idiographic component alongside criteriological diagnosis.

This comprehensive concept of diagnosis is implemented through the articulation of two diagnostic levels. The first is a standardised multi-axial diagnostic formulation, which describes the patient's illness and clinical condition through standardised typologies and scales... The second is an idiographic diagnostic formulation, which complements the standardised formulation with a personalised and flexible statement. [IDGA Workgroup, WPA 2003: 55]

Juan Mezzich, President of the WPA, describes comprehensive diagnosis as follows:

The emerging comprehensive diagnostic model aims at understanding and formulating what is important in the mind, the body and the context of the person who presents for care. This is attempted by addressing the various aspects of ill- and positive- health, by interactively engaging clinicians, patient and family, and by employing categorical, dimensional and narrative descriptive approaches in multilevel schemas. [Mezzich 2005: 91]

In this passage, Mezzich assumes that an idiographic element will be provided through narrative.

Writing in the journal Psychopathology, the psychiatrist James Phillips also equates narrative and idiographic elements and describes them both thus:

In the most simple terms, a narrative or idiographic formulation is an individual account with first-person and third-person aspects. That is, the patient tells her/his story, with its admixture of personal memories, events and symptoms, and the story is retold by the clinician. The latter's account may contain formal diagnostic, ICD10/DSM-IV aspects, as well as psychodynamic and cultural dimensions not found in the manuals. The clinician's account may restructure the patient's presentation, emphasizing what the patient didn't emphasize and de-emphasising what the patient felt to be important. It will almost certainly contextualise the presenting symptoms into the patient's narrative, a task which the patient may not have initiated on her own. Finally, the clinician will make a judgment (or be unable to make a judgment) regarding the priority of the biological or the psychological in this particular presentation, and will structure the formulation accordingly... [Phillips: 2005: 182]

In the context of increasing emphasis on user centrality, one might object to the emphasis on the clinician's work in restructuring the patient's narrative. On the other hand, a second view 
on the significant components of a narrative might well be an important aid to a patient. But here the important element in Phillips' account is the idea that a narrative formulation and an idiographic formulation are one and the same thing and hence, because comprehensive diagnosis includes narrative elements, it also includes idiographic elements.

This assimilation of narrative and idiographic is just what I wish to question. I will argue that idiographic understanding, construed as individualised judgement, is a myth. But why should one think that one wants to add an idiographic element - in a sense I will contrast with narrative - to conventional criteriological diagnosis? A nice statement of the motivation was provided in a referee's comment on an earlier draft of this paper.

[T] he real problem in diagnosis comes when the nomothetic is given too much emphasis. Time and time again what is required is something like the idiographic. Time and time again the categorical, pigeon-holing, approach to diagnosis has to be bent in order to accommodate the individual account. The question is merely how to characterise such an account.

According to this common view, psychiatry has too often been guilty of pigeon-holing its subjects by attempting to subsume them under general categories. What is needed instead is a reaction against such categorisation and idiographic judgement seems to provide just that. The problem, however, is to characterise just what idiographic judgement might be. Is it really distinct from existing criteriological diagnostic elements? What does idiographic understanding comprise?

\section{Back to Windelband}

To address these questions I will turn to the philosopher who first introduced these terms to psychology and psychiatry, Wilhelm Windelband [Lamiell 1998]. Windelband introduced the distinction between idiographic and nomothetic in his rectorial address in 1894. Having explained the supposed methodological, as opposed to substantial, nature of the distinction, my purpose is to examine three possible interpretations of it:

1. as a practical distinction of concrete methods;

2. as reflecting separable elements of a metaphysical divide between the particular and the general; and

3. as merely an abstraction from a unified model of judgement.

I will argue that none is a tenable account of an idiographic approach to psychiatry and that adding idiographic elements would threaten the validity of psychiatric diagnosis.

Windelband introduces his distinction by contrasting it with a distinction of subject matter.

At present, a certain classification of disciplines which attempts to establish knowledge of reality is regularly employed. They are distinguished into natural sciences [Naturwissenschaften] and sciences of the mind [Geisteswissenschaften]. Stated in this particular form, I regard the dichotomy as unfortunate. Nature and mind is a substantive dichotomy... not equivalent to a dichotomy based on modes of cognition. [Windelband 1980: 173]

Windelband argues that such a distinction is a hostage to the fortune of a metaphysical distinction of kind between mind and the rest of nature. In psychiatry, the interplay of both broadly psychological methods and neurology makes drawing such a distinction premature and unhelpful. Furthermore, it disguises the very significant similarities of method between at least some aspects of psychology or psychological psychiatry and other natural sciences.

What is the source of the methodological relationship between psychology and the natural sciences? It evidently lies in the consideration that both psychology and the natural sciences establish, collect, and analyze facts only from the viewpoint and for 
the purpose of understanding the general nomological relationship to which these facts are subject. [Windelband 1980: 174]

Instead of a distinction based on the subject matter of study, Windelband proposes a methodological distinction which places psychology (as he understands it) and other natural sciences on one side and other disciplines, which in Germany at the time were called 'sciences of the mind' but which have a distinct method, on the other. This gives rise to a characterisation of what he will call 'idiographic' as follows:

[T] he majority of the disciplines that are usually called sciences of the mind have a distinctively different purpose: they provide a complete and exhaustive description of a single, more or less extensive process which is located within a unique, temporally defined domain of reality. [Windelband 1980: 174]

\section{Is the distinction a practical distinction?}

As first introduced, idiographic understanding concerns individual or unique cases. But, given that the distinction is supposed to be at the level of method not substance (including a very general notion of substance), this is not fixed by the subject matter - a subject matter of individual cases, for example - so much as how that subject matter is approached. This methodological distinction is made clearer in the following passages in which the term 'idiographic' is first introduced:

At this point, we have before us a purely methodological classification of the empirical sciences that is grounded upon sound logical concepts. The principle of classification is the formal property of the theoretical or cognitive objectives of the science in question. One kind of science is an inquiry into general laws. The other kind of science is an inquiry into specific historical facts. In the language of formal logic, the objective of the first kind of science is the general, apodictic judgment; the objective of the other kind of science is the singular, assertoric proposition...

In view of the foregoing considerations, we are justified in drawing the following conclusion. In their quest for knowledge of reality, the empirical sciences either seek the general in the form of the law of nature or the particular in the form of the historically defined structure. On the one hand, they are concerned with the form which invariably remains constant. On the other hand, they are concerned with the unique, immanently defined content of the real event. The former disciplines are nomological sciences. The latter disciplines are sciences of process or sciences of the event. The nomological sciences are concerned with what is invariably the case. The sciences of process are concerned with what was once the case. If I may be permitted to introduce some new technical terms, scientific thought is nomothetic in the former case and idiographic in the latter case. Should we retain the customary expressions, then it can be said that the dichotomy at stake here concerns the distinction between the natural and the historical disciplines. However we must bear in mind that, in the methodological sense of this dichotomy, psychology falls unambiguously within the domain of the natural sciences. [Windelband 1980: 175-6]

These passages suggest the following rough practical distinction. Nomothetic approaches are those that chart lawlike, or nomological, generalities. Their aim is to describe generalities. Idiographic understanding concerns individual cases described in non-general ways. Both are forms of empirical inquiry.

This distinction fits modern psychological usage influenced by Allport in which 'idiographic' is used to describe case-study based qualitative research by contrast with quantitative cohortbased research (although whether Allport's use of nomothetic accords with Windelband's is a matter of dispute [Lamiell 1998]). So far, however, it does not explain precisely the difference of method, rather than of subject matter, of idiographic approaches and thus precisely what new element is being called for in comprehensive diagnosis. 
The putative distinction receives some further elaboration in the suggestion that nomothetic understanding concerns processes that are repeated because it concerns their invariant underlying form. Idiographic understanding, by contrast, concerns singular or non-repeated events.

Consider... the subject matter of the biological sciences as evolutionary history in which the entire sequence of terrestrial organisms is represented as a gradually formative process of descent or transformation which develops in the course of time. There is neither evidence nor even a likelihood that this same organic process has been repeated on some other planet. In this case, the science of organic nature is an idiographic or historical discipline. [Windelband 1980: 176]

This example also reinforces the methodological nature of the distinction. The same phenomenon can be treated as manifesting a constant underlying form (with repeatable effects) susceptible of nomothetic analysis or, taken as a whole, it can be thought of as a lengthy but single event, capable of idiographic, historical understanding. But, whilst the opposition to nomothetic suggests a practical distinction of sorts, it is not yet clear what idiographic understanding actually comprises.

Before examining what Windelband himself says, it is worth asking whether a distinction could be framed in just the terms so far used. Jim Lamiell (in private correspondence) suggests a kind of pragmatic distinction. Idiographic understanding concerns individual cases described without concern for the generalizability of the terms of the description. The descriptions might, in fact, be generalizable, but the immediate concern of the investigator is not with that question.

The problem with this suggestion is twofold. First, as an interpretation of Windelband it does not fit well with his advocacy of philosophy as exploring the logic of forms of knowledge by contrast with the psychological state of the investigator [e.g. Windelband 1980: 170].

Secondly, if whether an element is idiographic or nomothetic depends merely on the attitude of the clinician, that would not justify the call to add idiographic elements to comprehensive diagnosis or explain what the elements themselves would add to diagnosis.

Windelband himself offers a number of unsatisfactory hints of an account of what idiographic understanding actually is. Taking history as his example, he says:

[H]istory seeks structural forms... [Windelband 1980: 178]

This does not, however, distinguish history from a natural scientific account of chemical structures couched in invariant nomological terms. He continues:

[I]n the historical sciences,... [thought] is devoted to the faithful delineation of the particulars... [Windelband 1980: 178]

Again, 'faithful delineation' simply adds the idea that idiographic understanding involves accurate description. But of what sort? In fact, the main positive account involves a simile with the work of creative artists:

The historian's task... is to breathe new life into some structure of the past in such a way that all of its concrete and distinctive features acquire an ideal actuality or contemporaneity. His task, in relation to what really happened, is similar to the task of the artist, in relation to what exists in his imagination. This is the source of the relationship between historical accomplishment and aesthetic creativity, the kinship between the historical disciplines and belles lettres. [Windelband 1980: 178]

The comparison of research in the natural sciences and history will establish even more clearly the predominance of abstraction in natural science and of perceptuality in history... [T] he ultimate aim of history is always to extract and reconstruct from the raw material of history the true shape of the past in robust and vital clarity. History produces images of men and human life in the total wealth and profusion of their 
uniquely peculiar forms and with their full and vital individuality preserved intact.

[Windelband 1980: 179]

In these passages, Windelband suggests that idiographic understanding consists in a particularly vivid portrayal, or imaginative recreation, of individual events. This may seem to specify a practically applicable distinction. Furthermore, it hints at the role of imagination in empathy which Jaspers argued lay at the heart of psychiatry. In fact, however, it merely postpones the question of how imaginative recreation is supposed to be a form of understanding.

To see that imaginative recreation is not, itself, a form of understanding, consider how one might, for example, imaginatively recreate a situation for which one had not the least understanding of its meaning or significance. One might fabricate chess pieces and a chess board and recreate the closing moments of the 11 July 1972 Fischer Spassky chess game without understanding the role of the pieces, the board, the rules or strategy or even that it was a game. This would not amount to historical understanding. Thus imaginative recreation cannot be used to specify a novel idiographic element in a model of comprehensive diagnosis. (This suggests that there is more to empathy than mere imaginative recreation of another's mental states. I will return to this point later.)

Even taking the opposition of 'perceptuality' and abstraction seriously raises rather than answers questions: what is the relation supposed to be between perception, shorn of its nomothetic elements, and understanding? I will examine this point in the next section.

\section{Does the distinction reflect an underlying metaphysical divide?}

While contemporary use suggests that idiographic and nomothetic forms of understanding are practically distinguishable but not fundamentally distinct, Windelband suggests that his distinction goes deeper. He relates it to a fundamental metaphysical divide:

[T]his distinction connects with the most important and crucial relationship in the human understanding, the relationship which Socrates recognized as the fundamental nexus of all scientific thought: the relationship of the general to the particular. [Windelband 1980: 175]

Note first that if the relation between idiographic and nomothetic reflects the relation between particular and general, often called the 'problem of universals' in philosophy, then it cannot also correspond to that between history and scientific psychology. Both historical and scientific psychological disciplines apply general concepts to individual cases and so neither can be identified wholly with one or other side of a distinction between general and particular understood in this fundamental way.

Nevertheless, Windelband may intend to refer to a distinction which is gestured at by the practical distinction between history and scientific psychology but which is better understood as reflecting the relation between judgements about individuals and judgements of general kinds. To say this is to place him, a post-Kantian philosopher, in the context of a Kantian problem. I will, however, summarise the problem in contemporary terms.

Consider how, as a result of an experience of it, a subject correctly applies a concept to an object. They say, for example, of an object $a$ that it has the property $F$. Such a judgement subsumes an individual object under a general concept: general because it could in principle apply to other objects (whether or not in practice the object is unique in respect of $F$ ). What, however, guides the speaker in applying $F$ to $a$ ? Imagine that the subject grasps a rule which states that if any object $x$ has features $G$ then it can be subsumed under empirical concept $F$. The problem, however, returns at this level. What guides the subject in applying $G$ to $a$ ? It seems that any general rule of concept application will replicate the original problem.

Kant touches on this problem in the 'schematism' chapter of the first Critique. The main question of that chapter is how to apply what he calls the 'categories' - the hierarchy of very general and fundamental concepts in Kant's philosophical system - to experience. But he also 
deals with everyday empirical concepts. The categories present additional problems because as high level concepts - and unlike empirical concepts - they are 'heterogeneous from empirical intuitions' and an intermediary has to be provided to guide their application. Things are not as complicated in empirical cases. Nevertheless, even here Kant suggests that an intermediary is needed between concepts and objects:

[I]t is [still] schemata, not images of objects, which underlie our pure sensible concepts... The concept of 'dog' signifies a rule according to which my imagination can delineate the figure of a four footed animal in a general manner, without limitation to any single determinate figure such as experience, or any possible image that I can represent in concreto, actually presents. [Kant 1929: 182-183]

This does not answer the problem, however. Firstly, it is not clear what the figure or perhaps image of a 'four footed animal in general' might be like. Secondly, for any general schematic figure, the question of what determines that it applies to any particular dog would return. Kant recognises that this account does not really address the problem, commenting:

[T]his schematism of our understanding, in its application to appearances and their mere form, is an art concealed in the depths of the human soul, whose real modes of activity nature is hardly likely ever to allow us to discover. [ibid: 183]

If an account couched in general terms - such as general rules for applying concepts - merely replicates the problem, what solution is there? Perhaps, somehow, judgements driven by particulars themselves shorn of general conceptual elements underpin empirical judgement. If so, this might provide a route to explicating idiographic understanding as deriving from a fundamental metaphysical problem about concept application.

There is some evidence that Windelband regards emphasising idiographic understanding as a reaction against an over-emphasis on the general in the metaphysics of thought:

The commitment to the generic is a bias of Greek thought, perpetuated from the Eleatics to Plato, who found not only real being but also real knowledge only in the general. From Plato this view passed to our day. Schopenhauer makes himself a spokesman for this prejudice when he denies history the value of a genuine science because its exclusive concern is always with grasping the specific, never with comprehending the general... But the more we strive for knowledge of the concept and the law, the more we are obliged to pass over, forget, and abandon the singular fact as such...

In opposition to this standpoint, it is necessary to insist upon the following: every interest and judgment, every ascription of human value is based upon the singular and the unique... Our sense of values and all of our axiological sentiments are grounded in the uniqueness and incomparability of their object. [Windelband 1980: 181-2]

The suggestion here is that examining value judgements helps to reveal the fundamental importance of singular cases as opposed to general kinds in our judgements about the world. This implies that there is an important role for a kind of judgement in which there is no implicit comparison - as there is with any general concept - with other cases. Such a judgement would be essentially particular and thus could not include general conceptual elements.

Windelband's fellow neo-Kantian Heinrich Rickert also argues that value judgements are essentially particular or individualised. Unlike nomothetic accounts of, for example, the forces acting on bodies which are described and explained in general terms, judgements about the value of things are essentially singular or individualised judgements.

We are concerned here with the connection of objects with values; for a generalizing approach the objects are free of value-connection, they are exemplars, replaceable... This is what happens when we free the object of all 
connection with our interests - it becomes a mere exemplar of a general concept. An individualising approach is necessarily connected with the value-bound grasp of the object [mit der wertverbindenen Auffassung der Objekte]... [Rickert 1907: 354-5]

But such an idea needs further augmentation if it is not merely to beg the key question in assuming that a thing's value is not codified in general terms in the way its weight is.

This construal of idiographic suggests an immediate problem. How can an essentially one-off judgement meet a general aim of medical diagnosis: validity? What sense is there to the idea that a judgement that does not make implicit comparison to other cases is a valid judgement? I will approach this through a more familiar philosophical charge. If idiographic understanding is modelled on judgements of individuals shorn of reference to generals then it is an instance of what the US philosopher Wilfrid Sellars (1912-1989) calls the 'Myth of the Given'.

Sellars uses this phrase to characterise a form of foundationalism based on experiences that ground, but do not themselves depend on, other conceptualised beliefs.

One of the forms taken by the Myth of the Given is the idea that there is, indeed must $b e$, a structure of particular matter of fact such that (a) each fact can not only be noninferentially known to be the case, but presupposes no other knowledge either of particular matter of fact, or of general truths; and (b) such that the noninferential knowledge of facts belonging to this structure constitutes the ultimate court of appeals for all factual claims - particular and general - about the world. It is important to note that I characterized the knowledge of fact belonging to this stratum as not only noninferential, but as presupposing no knowledge of other matter of fact, whether particular or general. [Sellars 1997: 68-9]

Sellars goes on to accept that experience can provide non-inferential knowledge and that such experience can constitute the ultimate court of appeals for factual claims. But he denies the claim that they presuppose no other knowledge of particular matters of fact. The reason for denying this third claim is that Sellars takes there to be a dual dependence between the kind of knowledge expressed in perceptual reports and an overall world-view. He suggests that perceptual knowledge has to jump two hurdles. The first concerns the reliability of the perceptual report.

The second hurdle is, however, the decisive one. For we have seen that to be the expression of knowledge, a report must not only have authority, this authority must in some sense be recognized by the person whose report it is. And this is a steep hurdle indeed. For if the authority of the report "This is green" lies in the fact that the existence of green items appropriately related to the perceiver can be inferred from the occurrence of such reports, it follows that only a person who is able to draw this inference, and therefore who has not only the concept green, but also the concept of uttering "This is green" -- indeed, the concept of certain conditions of perception, those which would correctly be called 'standard conditions' -- could be in a position to token "This is green" in recognition of its authority. In other words, for a Konstatierung [perceptual report] "This is green" to "express observational knowledge," not only must it be a symptom or sign of the presence of a green object in standard conditions, but the perceiver must know that tokens of "This is green" are symptoms of the presence of green objects in conditions which are standard for visual perception. [Sellars 1997: 74-5]

Sellars' point here is that for a perceptual report (or for the perceptual experience reported) to have authority it must not only be a reliable indicator of the state of the world but also its subject must know that it is reliable. Such knowledge makes the report depend more generally on the subject's worldview. The report cannot underpin an individualised judgement.

More recently, John McDowell has proposed a much shorter argument against the Myth of 
the Given [McDowell 1994]. Empirical judgements are supposed to be rationally constrained by the state of the world. That is how beliefs as a whole are rationally answerable to the world. But the only model we have of a rational reason for a belief is a relation in which both items related are already conceptualised. (The paradigmatic reason for a belief is another belief.) So if the final step in giving a reason for an empirical belief is a judgement which points beyond the realm of concepts - as idiographic judgements work on this interpretation it will not sustain a rational friction between belief and the world.

Thus, if idiographic understanding is understood to be a direct response to a unique and incomparable object, as Windelband suggests, and if that response can be abstracted away from, or is prior to, a conceptualised judgement, then it cannot rationally influence judgement. If so, it cannot be a form of valid understanding and certainly not a kind of understanding that might be added to a model of comprehensive diagnosis in psychiatry which aims at validity.

\section{Is the distinction really an abstraction from a unified model of judgement?}

Windelband asserts the independence of idiographic and nomothetic forms of understanding. It is implied in the examples of history and scientific psychology. But it is also explicitly stated in passages such as:

[I]n the total synthesis of knowledge, which is the ultimate aim of all scientific research, these two cognitive moments remain independent and juxtaposed.

[Windelband 1980: 183 italics added]

Given, however, the difficulty in extracting a genuine distinction either from his account of the 'perceptuality' of history or from the metaphysical divide between particular and general concept or universal, it is worth considering whether the distinction is better understood as an abstraction rather than an account of two independent ingredients. What would this be like?

Consider, as a related analogy, Kant's account of empirical experience. Empirical content is the result of the interplay of two faculties. These are the faculties of 'receptivity' and 'spontaneity' responsible, respectively, for intuitions - brute experiential intake - and concepts. Their interplay is described in the slogan: "Thoughts without content are empty, intuitions without concepts are blind" [Kant 1933: 93, A51, B75]. But this is not to say that empirical experience can be decomposed into two independent factors: brute experiential intake and subsequent imposition of concepts. Again, as McDowell explains, any model of empirical judgement which starts with such a bare given will sever the rational connection between judgement and the world because the only model we have of a reason for a belief is a relation in which both items related are already conceptualised. Thus, while the Kantian slogan describes the dual dependence of judgement on experiential intake and concepts, neither plays a separable role.

In fact the idea that idiographic and nomothetic elements are not practically separable is expressed towards the end of the rectorial address where he suggests that history also essentially contains nomothetic elements:

[G]eneral propositions are necessary at every stage of inquiry in the idiographic sciences. And these they can borrow only - with perfect legitimacy - from the nomothetic disciplines. Every causal explanation of any historical occurrence presupposes general ideas about the process of things on the whole. When historical proofs are reduced to their purely logical form, the ultimate premises will always include natural laws of events, in particular, laws of mental events or psychological processes. Consider someone who has no idea at all concerning how men in general think, feel, and desire. It would not only be impossible for him to comprehend individual happenings in order to acquire knowledge of events and processes. He would already have failed in the critical determination of historical facts. [Windelband 1980: 182-3]

If this is what is meant by the idiographic/nomothetic distinction, however, neither aspect can 
provide a novel element to be added into comprehensive psychiatric diagnosis.

\section{Individualised idiographic judgement is a myth}

I have considered three ways of interpreting Windelband's distinction between idiographic and nomothetic understanding. It might be a practical distinction between concrete methods employed in history and scientific psychology (to take two examples). But the account actually offered of historical methods does not succeed in articulating a genuine form of understanding. It might, secondly, be interpreted as a distinction of independent factors in a long-standing fundamental distinction between particulars and general kinds or universals. On this interpretation, idiographic judgement would be deployed as part of an attempt to explain how it is possible to frame judgements about particulars rather than general kinds. But if so, the model of idiographic understanding is guilty of subscribing to the Myth of the Given and it cannot account for the rational connection between judgement and the world. It thus undermines the validity of psychiatric diagnosis or formulation. Or, thirdly, it might be interpreted not as a distinction between two separable ingredients within judgement but an abstraction of essentially combined ingredients. If so, however, it does not serve to outline a method of improving psychiatric diagnosis by adding a distinct element to conventional criteriological diagnosis.

Given the call for comprehensive diagnosis to include idiographic elements, there is no clear account drawn from Windelband as to what this novel element might be. Furthermore, the idea of one-off idiographic elements threatens the validity of psychiatric diagnosis.

\section{Narrative versus nomological understanding}

At the start of this paper I quoted both Juan Mezzich and James Phillips calling for idiographic elements to be included in a broader notion of psychiatric diagnosis or formulation. Both run together 'idiographic' and 'narrative'. I suggested that the motivation for calling for idiographic judgement was a reaction to the worry that psychiatry sometimes pigeon-holes its subjects under general nomothetic categories. Idiographic is the supposed cure for over-emphasis on nomothetic elements. But I have argued that Windelband does not succeed in drawing a distinction of kind between nomothetic and idiographic judgement. In this final section I will thus turn instead to second element in Mezzich's and Phillips' accounts: narrative. Whilst narrative judgement is tied to the kind of person-centred focus of contemporary psychiatric care it is not tied to the uniqueness of subjects as most definitions of the idiographic are [cf Lamiell 1997: 125-9]. I will suggest that this familiar distinction drawn from the social sciences is more successful than that between idiographic and nomothetic in explaining how a broader notion of psychiatric formulation - rather than a narrower notion of criteriological diagnosis - should be framed.

Narrative judgement contrasts with nomological or lawlike understanding or explanation not because it aims at one-off events but because it is normative. The appropriate contrast is not between the generality of nomothetic approaches and individualised judgement of particulars but rather between non-normative and normative descriptions. Narrative judgements thus answer to a different kind of internal logic to non-normative nomological accounts. In the vocabulary John McDowell has developed from Sellars, they belong to the 'space of reasons' rather than the 'realm of law' [McDowell 1994].

Why are narrative judgements normative? Three considerations support this claim. Firstly, those individual elements within narrative accounts of subjects that correspond to propositional attitudes are normative. An expectation, for example, defines the event that would satisfy it; a belief defines the state that would make it true. In forming such a propositional attitude, a subject adopts a normatively characterised stance to how the world is or will be.

Secondly, the connections between elements of a narrative account of a subject reflect how a subject ought to think and act. They answer, in Donald Davidson's phrase, to the 'constitutive 
ideal of rationality' which has 'no echo in physical theory' [Davidson 1980: 223, 231]. They are, as Jaspers says of empathic understanding, ideally typically meaningful connections. A subject is compared to an ideal of good thinking. This contrasts with subsumption of events under natural laws in which that normative dimension of what ought to happen is missing.

Tailored to an understanding of persons, the elements of a narrative are mental states described in a rational pattern of propositional attitudes.

The concepts of the propositional attitudes have their proper home in explanations of a special sort: explanations in which things are made intelligible by being revealed to be, or to approximate to being, as they rationally ought to be. This is to be contrasted with a style of explanation in which one makes things intelligible by representing their coming into being as a particular instance of how things generally tend to happen. [McDowell 1985: 389]

(This characterisation reveals why empathy can be more than mere imaginative recreation, to which point I promised to return. It is imaginative recreation of thinking in accord with the normative pattern of ideally typical, meaningful connections. The appreciation of why one thought or reaction is supported by another is what makes empathy sufficient of an achievement to count as a form of understanding.)

Thirdly, anomalies are differently accounted for in normative and non-normative forms of understanding. If an explanation by subsumption under a natural law fails because observations of what has happened fail to fit the law then so much the worse either for the accuracy of observations or for the natural law. But this is not the case for narrative accounts where deviations from the standards of good reasoning can be accommodated as occasional lapses of judgement, perhaps explained by other aspects of the pattern which do accord with the standards. (One can, for example, form a mistaken belief for a good reason.)

Despite this difference, narrative accounts are nevertheless couched in general terms and consequently there is no tension between adding such elements to comprehensive diagnosis and aiming for its validity. Thus if the supposedly idiographic elements that, in addition to general criteriological or nomological elements, make up comprehensive diagnosis are really narrative in this sense then they do add something genuinely distinct without risk to validity. They add a rational, normative form of understanding that has a different underlying logic to explanation by subsumption under natural laws. But whilst they help to outline individual human subjectivity by furnishing accounts that can be tailored to individual subjects, this is not to deny their implicit generality. The concepts that form narrative accounts can be applied in different ways to different subjects providing that the narratives so formed are shaped in recognisable rational patterns.

\section{Conclusions}

The call for a broader notion of formulation to augment a narrow criteriological model of diagnosis in psychiatry has recently been couched as a call for idiographic elements to be included. I have argued that this is misleading. If the reason for invoking idiographic elements is as a reaction against the charge that psychiatry pigeon-holes its subjects, it might seem that individualised or particular, by contrast with general, judgement is called for. But examination of the notion of idiographic judgement in Windelband's seminal account suggests that the distinction between it and nomothetic judgement cannot be drawn. Idiographic judgement, construed as individualised judgement, is a myth.

On the other hand, narrative judgement does seem to provide a genuine addition to a narrow model of psychiatric criteriological diagnosis. Narratives are essentially normatively structured, reason-based accounts of individual thought and action. They are the perspective from which a subject, as a person, comes into view. But, unlike supposed individualised judgements, they are essentially general and thus there is no a priori argument against their validity. 


\section{Bibliography}

Davidson, D. (1980) Essays on Actions and Events, Oxford: Oxford University Press

Hurlburt, R.T. and Knapp, T.J. (2006) 'Münsterberg in 1898, Not Allport in 1937, Introduced the Terms "Idiographic" and "Nomothetic" to American Psychology' Theory \& Psychology 8: 5-22

IDGA Workgroup, WPA (2003) ‘IGDA 8: Idiographic (personalised) diagnostic formulation’ British Journal of Psychiatry, 18 (suppl 45): 55-7

Kant, I. (1929) Critique of pure reason London: Macmillan

Lamiell, J.T. (1997) 'Individuals and the differences between them’ in Hogan, R., Johnson, J. and Briggs, S. (eds) (1997) Handbook of Personality Psychology, New York: Academic Press

Lamiell, J.T. (1998) “"Nomothetic” and “idiographic”' Theory \& Psychology 8: 23-38

McDowell, J. (1985) 'Functionalism and Anomalous Monism' in LePore, E. and McLaughlin, B.P. (eds) Actions and Events: Perspectives on the Philosophy of Donald Davidson, Oxford: Blackwell

McDowell, J. (1994) Mind and World, Cambridge, Mass.: Harvard University Press

McDowell, J. (1998) 'The Woodbridge lectures, Having the world in view: Sellars, Kant, and intentionality' Journal of philosophy 95: 431-95

Mezzich, J.E. (2005) 'Values and comprehensive diagnosis' World Psychiatry 4: 91-92

Phillips, J. (2005) 'Idiographic Formulations, Symbols, Narratives, Context and Meaning' Psychopathology 38: 180-184

Rickert, H. (1907) 'Geschichtsphilosophie' in Windelband, W. (ed) Die Philosophie im Beginn des zwanzigsten Jahrhunderts: Festschrift für Kuno Fischer, Heidelberg: Carl Weinter, 1907: 321-422 (translation Lyne, I.)

Sellars, W. (1997) Empiricism and the Philosophy of Mind, Cambridge, Mass.: Harvard University Press

Windelband, W. (1980) 'History and natural science’ History and Theory \& Psychology 19: 169-85. 